Undercooked hamburgers contaminated with $E$. Coli 0157:H7 were responsible for a major outbreak of intestinal illness in 5 western states in 1993. Ground beef is unlike most other raw meats in that microbial contaminants are distributed throughout the product by grinding. Hamburger cooked rare or medium-rare can still contain viable organisms on the inside.

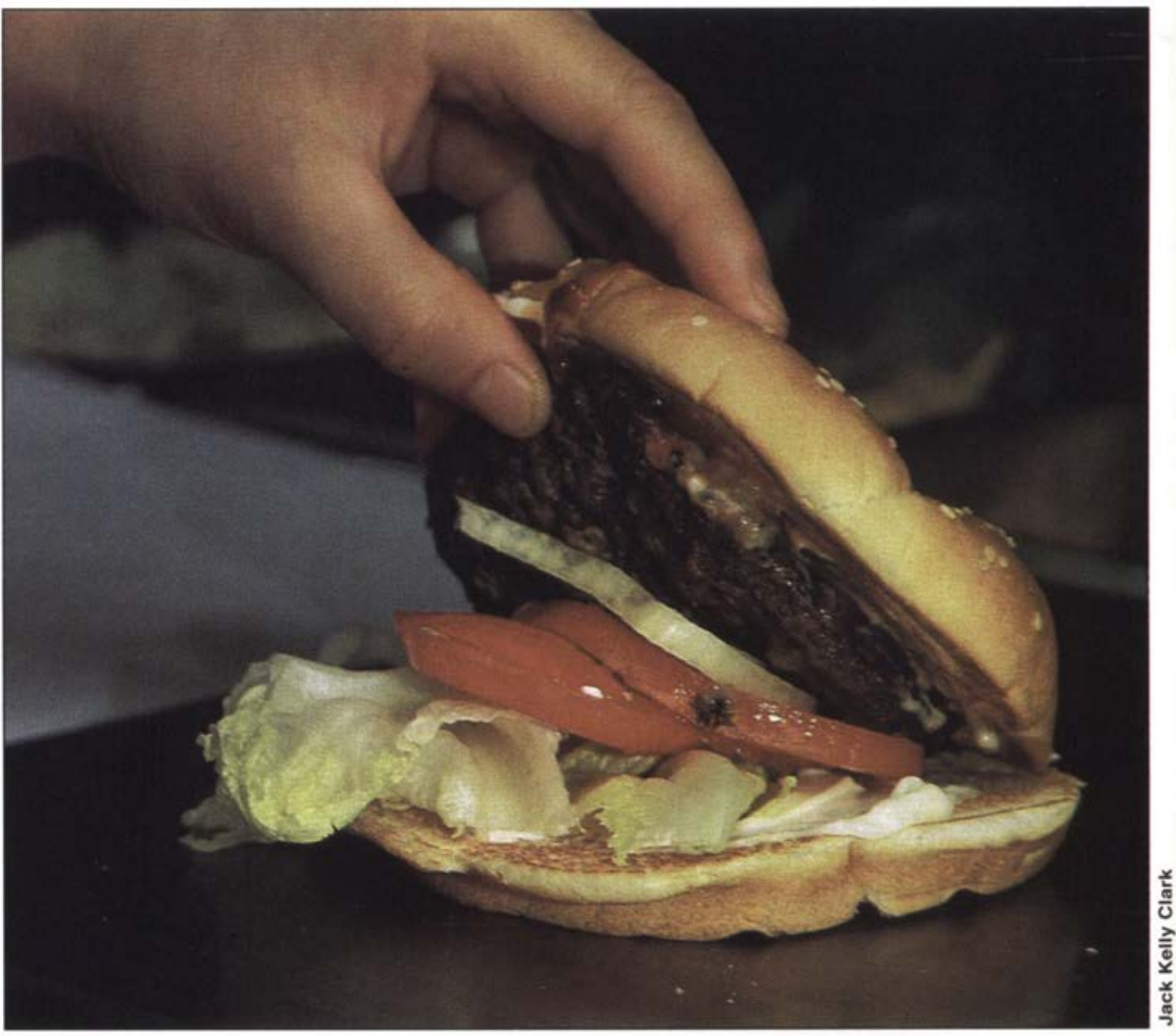

\title{
Research and reason can minimize foodborne and waterborne illnesses
}

\author{
Dean O. Cliver $\quad$ Edward R. Atwill
}

\author{
Several outbreaks of foodborne \\ and waterborne illness have di- \\ rected the nation's attention to in- \\ testinal pathogens that are threats \\ to public health. Among these \\ pathogens are Cryptosporidium \\ parvum and Escherichia coli \\ 0157:H7, which are known to in- \\ fect and to be spread by not only \\ humans, but also livestock and \\ various species of wildlife. New \\ regulations aimed at controlling \\ these pathogens are being imple- \\ mented, despite a shortage of sci- \\ entific information about their \\ ecology, how they contaminate \\ food and water supplies, and how \\ to detect and eliminate such con- \\ tamination. Research is needed to \\ address these issues and to de- \\ velop better technologies for \\ pathogen detection, water treat- \\ ment and food processing.
}

Recent outbreaks of foodborne and waterborne disease in the United States have heightened concerns about several specific pathogens and prompted actions by federal, state and local agencies that could have serious implications for agriculture. Two pathogens in particular - the protozoan Cryptosporidium parvum and the bacterium Escherichia coli O157:H7 have caused major disease outbreaks in the past few years.

- In April 1993, C. parvum contaminated the public water supply in Milwaukee, Wis., causing 403,000 cases of diarrhea and more than 100 deaths (MacKenzie et al. 1994). It was the largest outbreak of waterborne disease ever documented in the United States.

- In January 1993, an outbreak of intestinal illness first detected in Washington state was traced to undercooked fast-food hamburgers contaminated with the bacterium
E. coli O157:H7 (Bell et al. 1994). More than 700 people in five western states were made ill with bloody diarrhea or kidney failure, at least four died, and several survivors lost part or all of their large intestines or kidneys.

- In October 1996, another outbreak of illness caused by E. coli $0157: \mathrm{H7}$ was traced to fresh, unpasteurized apple juice. A total of 66 cases was reported from three states and British Columbia. One child died of kidney failure caused by the illness.

The 1993 outbreaks caused widespread public concern and prompted regulatory actions at federal, state and local levels. In 1996, the U.S. Department of Agriculture (USDA) announced a sweeping reform of federal food safety rules for meat and poultry, while the Environmental Protection Agency (EPA) issued new rules and amendments to the Safe Drinking Water Act. 


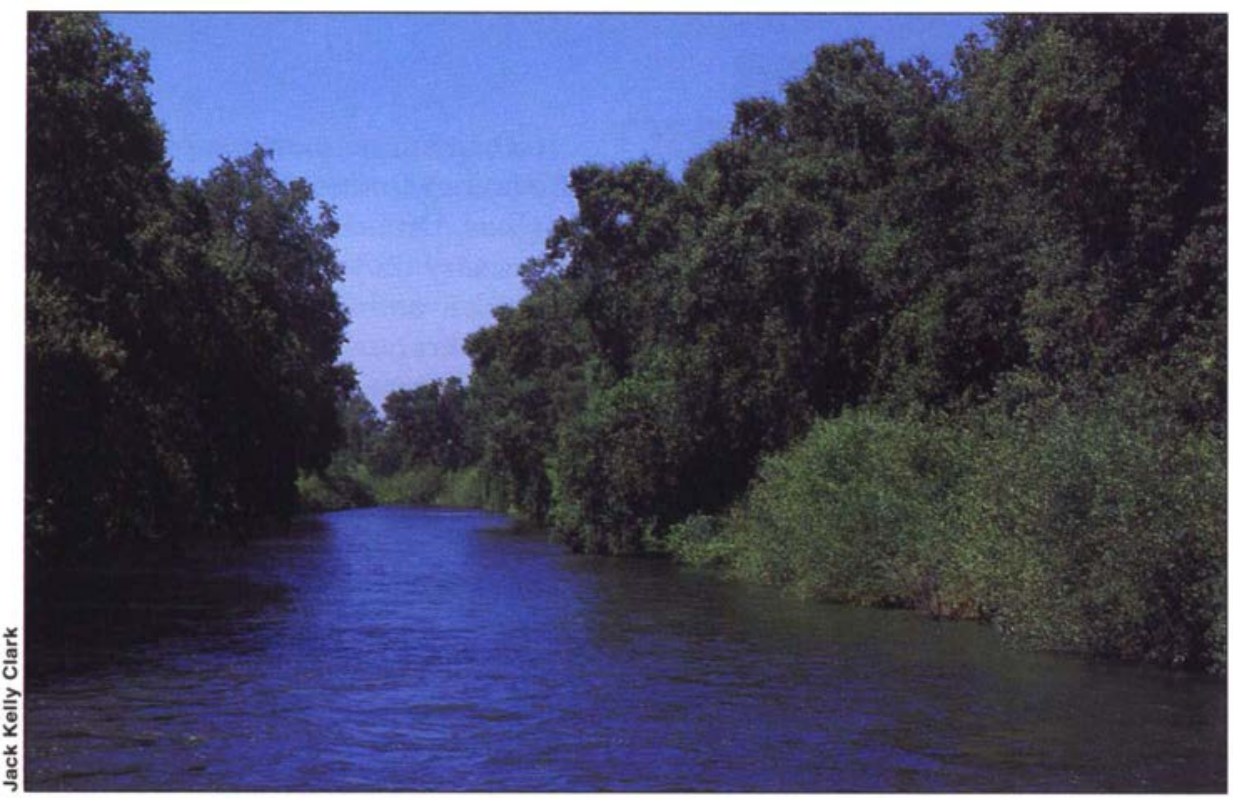

Surveys indicate that Cryptosporidium oocysts are common in surface waters throughout North America, but the limitations of current tests make it difficult to learn where significant hazards lie. Although drinking water is treated, no test is available that can rapidly and sensitively detect $C$. parvum in treated water.

The new regulations call for, among other things, increased sampling and testing and improved control measures for foodborne and waterborne pathogens. On a local level, some public water districts are considering changes in watershed management. In some cases, owing to the level of public concern, regulatory action has preceded scientific understanding. Little is known about the medical ecology of these organisms, their sources, and how best to detect and control them. Several studies, funded in part by the UC Division of Agriculture and Natural Resources, are now under way to address these issues.

\section{Common features}

Both E. coli O157:H7 and C. parvum infect the intestines and are shed in feces. E. coli $\mathrm{O} 157: \mathrm{H7}$ is a virulent strain of the harmless E. coli bacterium that normally inhabits the intestines of humans and other warm-blooded animals. This pathogenic strain is known to infect and to be shed by humans, cattle, sheep, deer and possibly other ruminants. C. parvum infects about 80 species of animals, including humans, cattle, sheep, goats, pigs, horses, dogs, cats and various wildlife species, such as deer, raccoons, opossums, pigs and rabbits. Both organisms have been found throughout the world, wherever researchers have looked for them.
These pathogens can spread in a variety of ways, but always through fecal matter. They may pass directly from host to host (e.g., among children in day-care centers or among young animals in a holding pen), as well as via fecally contaminated food and water. Although people usually defecate in toilets, if they don't wash their hands carefully afterward they may convey pathogens to food. Surface water can become contaminated from unintentional discharges of raw or inadequately treated sewage or from runoff of domestic and wild animal wastes.

In some outbreaks of foodborne or waterborne disease, the contamination has clearly had a human origin, such as a food handler with an intestinal infection or a swimmer shedding the pathogen into water. There have been cases of both cryptosporidiosis and $E$. coli O157:H7 infection acquired from swimming in lakes, and some cases of cryptosporidiosis have been linked to swimming pools.

The source of the contamination of Milwaukee's water supply, which is drawn from Lake Michigan, was never determined. Possible sources considered by investigators included cattle along two rivers that flow into the Milwaukee harbor, slaughterhouses, and human sewage (MacKenzie et al. 1994). Cattle have been implicated in several outbreaks of waterborne ill- ness, but the evidence has seldom been conclusive. Although we know that both $C$. paroum and E. coli O157:H7 can be shed by mammalian sources other than humans and cattle, such sources are rarely explored during outbreak investigations. Several UC researchers and others are working to develop tests that would make it easier to trace the source of contamination in such outbreaks.

Foodborne disease outbreaks caused by E. coli $\mathrm{O} 157: \mathrm{H} 7$ and similar agents have been associated with contaminated ground beef, roast beef, dry Italian-style salami, raw milk, apple juice, cantaloupe, lettuce and other vegetables. Ground beef is the most commonly implicated vehicle of transmission in foodborne outbreaks of E. coli O157:H7 infection (Armstrong et al. 1996). Beef may become contaminated if intestinal contents contact the meat during slaughter or processing. Bacteria present on the surface of the meat are distributed throughout the meat by grinding.

\section{Foodborne vs. waterborne: What's the difference?}

Whether a pathogen is called foodborne or waterborne depends on its primary vehicle of transmission. E. coli O157:H7 is primarily foodborne - but people have been sickened by this microbe after swimming in contaminated lakes. Fewer than $\mathbf{5 0}$ E. coli $\mathrm{O} 157: \mathrm{H} 7$ bacteria can cause serious illness. Fortunately, this pathogen is destroyed by chlorine and so is usually not a problem in drinking water.

E. coli 0157:H7 presents greater food risks than $C$. parvum. Specifically, E. coli O157:H7 can continue to multiply outside human or animal hosts, even on refrigerated meat. Cooking meat to an internal temperature of $155^{\circ} \mathrm{F}$ kills this pathogen.

The history of outbreaks suggests that C. parvum is a greater risk in drinking water than in food, largely because it is not killed by chlorine. It can be transmitted on food, but it does not proliferate outside human or animal hosts. Its oocysts remain dormant until ingested by a human or animal host. While as few as 30 oocysts can cause illness, it is self-limiting in healthy individuals, lasting for a few days to 2 weeks. However, it can be life-threatening to those with weakened immune systems. 


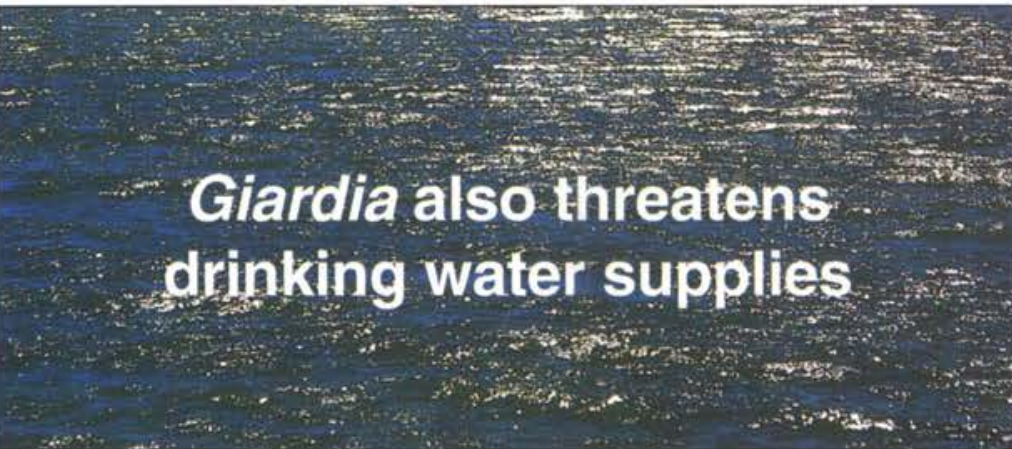

The intestinal protozoan Giardia is a leading cause of waterborne illness in humans. Like Cryptosporidium, Giardia has a simple life cycle, alternating between an active form that colonizes the intestines of the host and causes diarrhea, and resistant cysts that are passed from the body in the feces. The cysts can survive for long periods in the environment and cause infection when ingested by another host. Unlike Cryptosporidium, there are effective drugs for treating Giardia infection.

More than 100 outbreaks of waterborne giardiasis have occurred in the United States since 1965. Most of these outbreaks have been attributed to contamination of surface water with human sewage. Giardia is also commonly spread by direct person-to-person, fecaloral transfer of cysts, and giardiasis outbreaks have been associated with poor hygiene or inadequate sanitary conditions in day-care centers, nurseries and public institutions. Foodborne transmission from infected workers or family members can also occur.

Although Giardia infection is common in a wide variety of wildlife and livestock species, there is considerable controversy over the issue of whether cysts shed by animals can infect humans. Investigators have been unable to determine conclusively the host origin of Giardia cysts detected in the majority of waterborne outbreaks.

The evidence for zoonotic transmission of giardiasis from animals to humans is circumstantial. Giardia isolates from humans have been experimentally transmitted to other animals, including beavers and muskrats. In the wild, the reported prevalences of Giardia infection in these aquatic mammals are 7 to $16 \%$ for beavers and over $95 \%$ for muskrats, but the Giardia species isolated from these animals appear to be different from those that infect humans.

Sheep, goats, pigs, horses and cattle have also been implicated as potential sources for transmission of giardiasis to humans. We have found up to $27 \%$ of California range beef cattle to be shedding Giardia in their feces, with the majority of infections occurring in calves. We also detected shedding of Giardia in 4 to $6 \%$ of California horses. Despite the isolation of Giardia from these hosts, there exists considerable controversy among researchers as to whether Giardia from livestock is infectious to humans under natural conditions.

Taxonomic confusion is a major impediment to resolving the question of zoonotic transmission. Some investigators have named Giardia species based on their host origin, while others have based species designations on morphological features of the protozoans. Currently, researchers are using various molecular analyses to distinguish different species. It remains unclear, however, how many species of Giardia infect humans or what species are involved in waterborne outbreaks.

Just as Cryptosporidium oocysts are ubiquitous in surface water throughout North America, Giardia cysts are also found in a majority of surface water samples. As with Cryptosporidium oocysts, however, available tests cannot determine whether the detected cysts are infectious to humans. From a practical standpoint, given the scientific uncertainties, any Giardia cysts found in water must be considered potentially infectious to humans. Giardia cysts can be removed by filtration and are not as resistant to chlorine-based disinfectants as Cryptosporidium oocysts. -Tim Stephens
It is not known how people typically become infected with Cryptosporidium. Drinking water was clearly the primary transmission vehicle in Milwaukee and in other major outbreaks of cryptosporidiosis. In general, however, the relative importance of various possible transmission routes is unclear. Food-related outbreaks have involved raw milk, possibly raw sausage, tripe, kefir and apple juice. During the Milwaukee outbreak, Cryptosporidium carried in tap water was suspected of contaminating salad dressings, dairy products and sausages, so the suspect foods were recalled.

\section{IIIness in humans}

No drug is known to be effective in treating the infections and illnesses caused by these pathogens. $E$. coli O157:H7 and several other, less common strains of $E$. coli produce a toxin similar to Shiga toxin of Shigella dysenteriae and cause severe bloody diarrhea in humans. The infection can lead to kidney failure (hemolytic uremic syndrome) and death.

Shiga toxin-producing E. coli are notably more resistant to acid conditions (as in apple juice) than nonpathogenic strains of E. coli. E. coli O157:H7 can survive a $\mathrm{pH}$ as low as 2.0 under certain circumstances (Armstrong et al. 1996). The bacteria can multiply in foods and elsewhere in the environment under some conditions, as well as in the host's body. They can be killed by heat, chlorine or UV light. An internal temperature of $155^{\circ} \mathrm{F}$ during cooking is sufficient to kill more than $99 \%$ of organisms in ground beef (Bell et al. 1994).

The largest documented outbreak of foodborne illness caused by $E$. coli O157:H7 occurred in Japan in 1996, when more than 9,000 people became ill and at least 11 died. The source of the outbreak was never determined.

Cryptosporidium infection causes watery diarrhea associated with abdominal cramping, nausea, vomiting and fever. In people with normal resistance, cryptosporidiosis is a selflimiting illness from which most patients recover after a few days or weeks. Those with compromised immune systems, however, develop 
long-term infections and may die from the illness. People at greatest risk from cryptosporidiosis include organ transplant recipients, AIDS patients and some cancer patients.

Cryptosporidium is transmitted among hosts in the form of "oocysts," which cannot multiply in foods or elsewhere in the environment. The oocysts are very resistant to chlorine but probably not to heat or UV light. Each oocyst contains four "sporozoites" that emerge and infect a new host's intestines after the oocyst is swallowed. There are several species of Cryptosporidium that infect animals, but only C. parvum is known to infect humans.

Studies indicate that very few viable cells of $E$. coli O157:H7 or oocysts of $C$. parvum need to be ingested with food or water to produce infection. For example, an infectious dose of 132 calfderived oocysts caused cryptosporidial infections in $50 \%$ of healthy human volunteers (Dupont et al. 1995). The lowest infectious dose sufficient to induce cryptosporidiosis was 30 oocysts. In outbreaks of $E$. coli 0157:H7 infection, the infectious dose has been estimated to be fewer than 50 bacteria and, in some cases, as few as 5 (Armstrong et al. 1996).

\section{Regulatory actions}

In response to the 1993 disease outbreak caused by $E$. coli O157:H7 in fast-food hamburgers, the Clinton administration mandated safe-handling labels for raw meat and poultry products, declared E. coli O157:H7 an adulterant in raw ground beef (making the sale of contaminated meat unlawful) and initiated a testing program for that pathogen. Ground beef is unlike most other raw meats in that microbial contaminants are distributed throughout the product by grinding, rather than being concentrated on the surface. As a result, a hamburger cooked rare or medium-rare (as preferred by about one-fourth of the U.S. population) still contains viable organisms on the inside. This is a particular concern given the extremely low infectious dose of E. coli O157:H7.

The 1993 outbreak also set in motion a major revision of the USDA's food safety program, culminating in

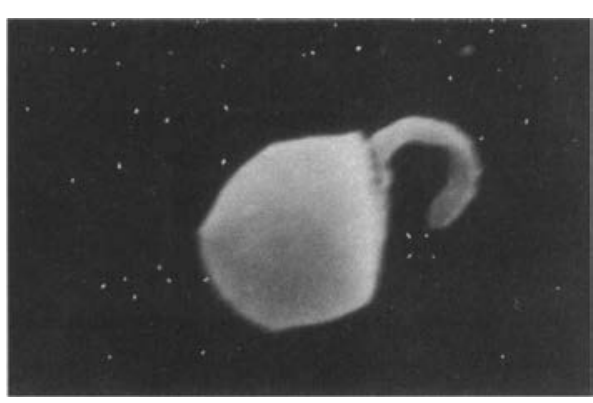

Each Cryptosporidium oocyst, about 4-micrometers in diameter, contains four sporozoites.

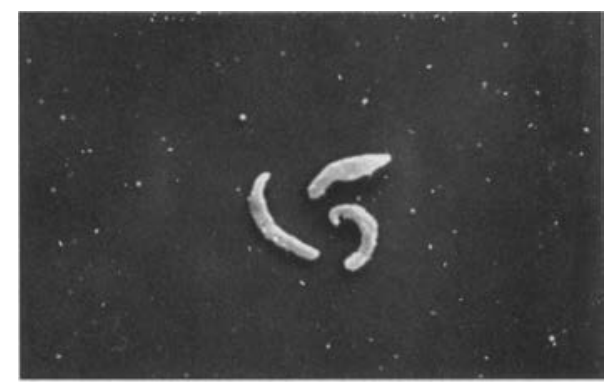

Cryptosporidium sporozoites infect a host's intestines, causing watery diarrhea, abdominal cramping, nausea, vomiting and fever.

the 1996 reforms known as the "Final Rule on Pathogen Reduction and Hazard Analysis and Critical Control Point (HACCP) Systems" (USDA 1996). The new regulations require all slaughter and processing plants to implement HACCP plans. The HACCP (pronounced "hassip") concept originated in the space program to ensure food safety for astronauts. It requires food processors to identify "critical control points" where hazards such as microbial contamination can occur, establish controls to prevent or reduce those hazards, and maintain good records.

In addition, the new rules require slaughter plants to conduct microbial testing of carcasses for generic $E$. coli to verify that their procedures are working to prevent fecal contamination. New performance standards for Salmonella require slaughter plants and producers of raw ground meat to ensure that their Salmonella contamination rates are below the current national baseline. And finally, all plants must adopt standard operating procedures for sanitation.

Similarly sweeping reforms of the EPA's drinking water regulations

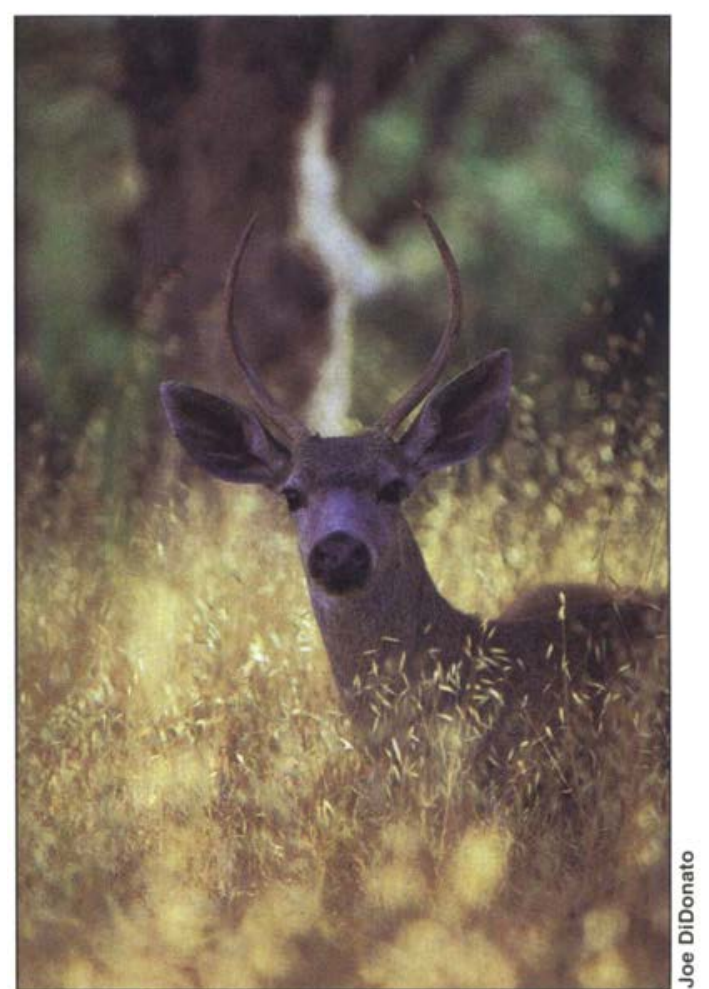

Wildlife, including black-tailed deer, may be significant sources of cryptosporidial contamination of surface water.

were at least partly inspired by the Milwaukee outbreak of cryptosporidiosis. Under proposed changes to the Surface Water Treatment Rule, public water systems that use surface water sources may be required to treat specifically for Cryptosporidium (EPA 1994).

Cryptosporidium oocysts are resistant to chlorine and may not be eliminated by disinfection procedures designed to remove other protozoan pathogens, such as Giardia. Even a well-run water-treatment system cannot ensure that drinking water will be completely free of the parasite. Because no available test can rapidly and sensitively detect Cryptosporidium in treated water, water-treatment plants can only monitor water-quality indicators such as turbidity and try to maintain a level of water quality (through filtering and other procedures) that makes the presence of oocysts unlikely (MacKenzie et al. 1994). The EPA may require some water systems using unfiltered surface water to implement filtration procedures.

The EPA has acknowledged that numerous scientific uncertainties must be resolved before it can establish 
prehensive food-safety strategy that addresses hazards at other points "from farm to table." This could eventually put the onus on farmers to eliminate pathogens from their herds, even though the means to do so may not be available.

When outbreaks of foodborne illness occur, regulatory efforts are often directed upstream, beginning with the distributor, leading back to the processor and, if possible, finally reaching the original producer. Pathogens can contaminate food products anywhere along the line from producer to consumer. In many cases, the contamination occurs at centralized processing facilities or from improper handling by consumers. For instance, foodborne bacterial pathogens can multiply from a harmless level to one high enough to cause illness in humans if the product is not refrigerated or is otherwise improperly handled.

If established management practices can eliminate a pathogen at the production level, there may be justification for holding producers responsible for its presence. However, there are many emerging foodborne pathogens about which we know almost nothing. In the case of E. coli O157:H7, for example, we have very little information about how the bacterium is introduced into a dairy or cow-calf operation or why it often appears to suddenly leave the herd.

While it is entirely reasonable that we expect food to be safe and wholesome, some consumers and public officials have expressed zero tolerance for risk. It will be extremely difficult, if not impossible, to implement public policy that will satisfy them. Society's growing detachment from how food is raised results in the unreasonable expectation that farms or ranches can be run as microbe-free, sterile environments. We often can raise and grow food with a minimum of pathogenic microorganisms on the product, but it is not a sterile commodity.

Both C. parvum and E. coli O157:H7 can be killed in food by irradiation, although this is not a widespread practice. The U.S. Food and Drug Administration does not permit irradiation of red meats.
The most generally applicable means of decontaminating foods is with heat, by pasteurization or cooking. Consumers must use proper food preparation and cooking practices to minimize foodborne disease. At the same time, producers and processors should adopt management practices that minimize the levels of pathogens present on the product. However, agriculture can only adopt such practices once they have been identified through research. Moreover, we know very little about the ecology of foodborne pathogens at the farm level.

\section{Research in progress}

Research is under way - in part funded by the UC Division of Agriculture and Natural Resources - to estimate the prevalence and to identify risk factors for shedding of a variety of possible foodborne bacterial and protozoal pathogens in livestock populations. If, for instance, we find that beef calves being produced by California's 700,000 to 950,000 beef cows are not significantly infected by major foodborne pathogens, then devising management practices that focus on cow-calf production will likely have little impact on reducing the incidence of foodborne illness associated with beef products. If, on the other hand, calves coming off of California's rangeland are an important entry point for a foodborne pathogen, then through research, extension and working closely with the industry we can develop management plans that minimize risk of foodborne disease.

Preliminary data suggest that less than $1 \%$ of rangeland beef calves are shedding E. coli $\mathrm{O} 157: \mathrm{H7}$ and an equally low proportion of calves are shedding C. parvum at time of weaning, 6 to 9 months of age. A more sensitive test for detecting E. coli $\mathrm{O} 157: \mathrm{H} 7$ probably would have found more infected cattle. What we cannot say at this time is whether these low prevalences of shedding pose a risk to the consumer and what is the relative importance of animal shedding postweaning, during stock operations, in the feedlot, or at the time of slaughter. How meat products are handled and refrigerated after slaughter directly affects the numbers of bacteria present on the finished product and may completely nullify any preharvest efforts directed at food safety.

It is not known whether most of the E. coli $\mathrm{O} 157: \mathrm{H} 7$ that occasionally contaminates beef carcass surfaces comes from the animal's intestines or from the hide. Much research - being conducted at several universities, including UC Davis, and USDA - is currently aimed at identifying critical control points at slaughter to decontaminate the surface of a carcass. Most methods being tested are based on washing the carcass surface with water or solutions containing organic acids, hypochlorous acid, hydrogen peroxide, or trisodium phosphate. Although several of these methods look promising, there is some doubt that any will guarantee the absence of pathogenic E. coli, generic E. coli, or Salmonella.

With respect to dairy cows and milk production, we find very few cows shedding C. parvum in their manure, but we cannot explain why we find C. paroum in the flush water coming from the wash pen for lactating cattle, nor can we say what level of shedding results in an appreciable risk of milkborne transmission. Does the widespread occurrence of $C$. parvum infection in dairy calves pose any risk to the veal market or to lactating cattle? The answers to these critical questions will show where on-farm or on-ranch problems exist - and just as important, where they do not exist. Only then can we devise effective solutions.

We and other researchers are also working to develop new detection methods for pathogens such as C. parvum. Better tests will help clarify where actual risks exist, allow us to evaluate different water-treatment and food-processing methods for inactivating pathogens, and help prevent false alarms resulting from detection of pathogens that are already dead because of food processing practices or other mechanisms. Finally, a group of UC researchers is taking a multidisciplinary approach to understand how oocysts travel through a watershed from land to water. 


\section{Research, education needed}

Recent outbreaks of foodborne infectious disease caused by $E$. coli O157:H7 and C. parvum have gotten the public's attention. Food safety solutions will come only with a detailed understanding of how and why pathogens enter the food supply; better detection and processing technologies; working cooperatively with producers, processors and distributors; and educating consumers through such means as UC Cooperative Extension on how to prepare and cook their food properly and safely.

D.O. Cliver is Professor, and E.R. Atwill is Extension Veterinarian and Assistant Professor, Department of Population Health and Reproduction, School of Veterinary Medicine, UC Davis.

\section{References:}

Armstrong GL, Hollingsworth $\mathrm{J}$ and Morris JG Jr. 1996. Emerging foodborne pathogens: Escherichia coli $0157: \mathrm{H} 7$ as a model of entry of a new pathogen into the food supply of the developed world. Epidemiol Rev 18(1):29-51.

Atwill, ER. 1996. Assessing the link between rangeland cattle and waterborne Cryptosporidium parvum infection in humans. Rangelands 19(2):48-51.

Bell BP, Goldoft M, Griffin PM, et al. 1994. A multistate outbreak of Escherichia coli O157:H7associated bloody diarrhea and hemolytic uremic syndrome from hamburgers: the Washington experience. JAMA 272(17):1349-53.

Dupont HL, Chappell CL, Sterling CR, et al. 1995. The infectivity of Cryptosporidium parvum in healthy volunteers. N Engl J Med 332:855-9.

[EPA] Environmental Protection Agency. 1994. National primary drinking water regulation: enhanced surface water treatment requirements. Federal Register $59(145): 38832-58$.

Klesius PH, Hayes TB and Malo LK. 1986. Infectivity of Cryptosporidium sp. isolated from wild mice for calves and mice. J Am Vet Med Assoc 189:192-3.

MacKenzie WR, Hoxie NJ, Proctor ME, et al. 1994 A massive outbreak in Milwaukee of cryptosporidium infection transmitted through the public water supply. N Engl J Med 331(3):161-7.

Snyder, DE 1988. Indirect immunofluorescent detection of oocysts of Cryptosporidium parvum in the feces of naturally infected raccoons (Procyon lotor). J Parasitol 74:1050-5.

[USDA] U.S. Department of Agriculture, Food Safety and Inspection Service. 1996. The final rule on pathogen reduction and hazard analysis and critical control point (HACCP) systems. Background Papers, July 1996

Webster, JP and McDonald, DW. 1995. Cryptosporidiosis reservoir in brown rats (Rattus norvegicus) in the U.K. Epidemiology and Infection 115:207-9.

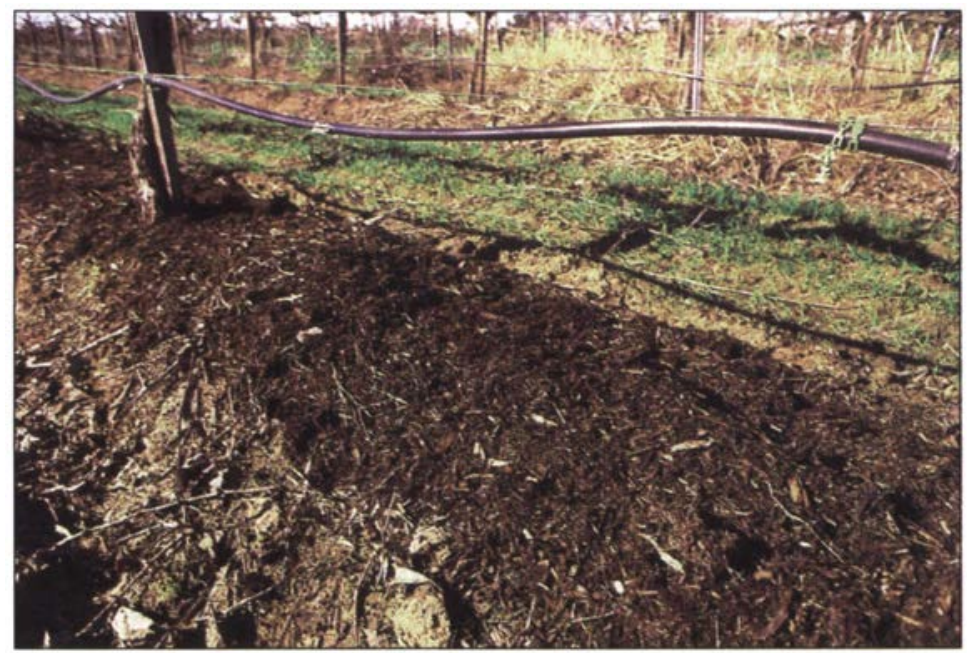

4 Composted "greenwaste" mulch was applied in the vine row for weed control. The wood chips cost more than the other treatments, but they controlled weeds well and half the chips were still in the vineyard after the second year.

\section{Mulch plus herbicides effectively control vineyard weeds}

\section{Clyde L. Elmore $\square \quad$ John Roncoroni $\square \quad$ Layne Wade Paul Verdegaal}

Mulches have been used for many years to control weeds by smothering the weed seedlings. A 2-year study in a Lodi grape vineyard compared the weed-control effectiveness of herbicides, cultivation, cover crop biomass and woodchip mulch and the cost of these practices. The most effective and least expensive treatment over the 2 years was the use of preemergence herbicides and a postemergence herbicide as needed. Growing cover crops, chopping the biomass and placing it into the vine row was very effective the second year, when more biomass was produced and weeds were controlled prior to mulch placement. The mulch was persistent in the field and should give long-term weed-control benefits, which were not evaluated in this study.
Weed management with herbicides in the vine row has been very successful for the control of annual weeds and some perennial weeds. However, weed species change as herbicides in different families are used, and unless different herbicides are used a weed species may become more severe than before herbicides were used. When annual weeds are controlled, some perennial weeds become more common and are more difficult to control. Herbicide costs have increased as new herbicides become available. In addition, there is interest in reducing pesticide inputs into the vineyard and in managing vegetation in the row by other methods, while at the same time growing cover crops between the vine rows.

Mulches have been used for many years to control weeds by smothering the weed seedlings. Mulches are commonly used in the landscape to suppress emergence and growth of weeds. At the same time that weeds are con- 